\title{
¿Son problemáticos para el veritismo la naturaleza y el valor del entendimiento?
}

\author{
Miguel Ángel FERNÁNDEZ \\ Instituto de Investigaciones Filosóficas \\ Universidad Nacional Autónoma de México \\ mafv@filosoficas.unam.mx
}

\begin{abstract}
Resumen: Este trabajo examina una serie de objeciones recientes a la epistemología veritista en el sentido de que no es capaz de explicar la naturaleza y el valor del entendimiento. La sección 1 formula las tesis centrales de una epistemología veritista y da algunos preliminares más. Las secciones 2 y 3 discuten objeciones que intentan mostrar que los estados cognitivos implicados en el entendimiento tienen una naturaleza sui generis a la que el modelo veritista de evaluación no puede aplicarse. Las secciones 4 y 5 examinan objeciones que intentan mostrar que hay aspectos del entendimiento que son epistémicamente valiosos y cuyo valor no puede explicarse en términos veritistas. La sección 6 resume los resultados de la discusión: ninguna de las objeciones examinadas consigue revelar algún déficit explicativo del veritismo con respecto a la naturaleza o el valor del entendimiento.
\end{abstract}

Palabras clave: epistemología veritista, valor epistémico, habilidad cognoscitiva, explicación, sistematicidad

\begin{abstract}
This paper examines a series of recent objections to veritistic epistemology that aim to show that it cannot account for the nature and value of understanding. Section 1 formulates the central tenets of veritistic epistemology and makes a few more preliminary remarks. Sections 2 and 3 discuss objections that attempt to show that cognitive states implicated in understanding have a sui generis nature which makes the veritistic model of evaluation inapplicable to them. Sections 4 and 5 examine objections that aim to show that there are aspects of understanding which are epistemically valuable, whose value cannot be explained in veritistic terms. Section 6 sums up results of the discussion: none of the objections discussed succeeds in revealing any explanatory deficit of veritism with respect to the nature and value of understanding.
\end{abstract}

Key words: veritistic epistemology, epistemic value, cognitive ability, explanation, sistematicity

\section{Introducción}

En años recientes se ha argumentado que el hecho de que la epistemología moderna se concentrara en enfrentar el escepticismo repercutió en que el espectro de logros cognitivos estudiados en esta área de la filosofía se estrechara y abarcara sólo el conocimiento y la justificación, en vista de que son éstos los logros que el escéptico pone en duda. 
Puesto que el conocimiento es conocimiento sólo de verdades, y la justificación es justificación para creer que algo es verdadero, la noción de creencia verdadera se convirtió en la piedra de toque en el estudio y la comprensión de los aspectos valiosos o exitosos de la cognición. Desde este punto de vista, la concentración obsesiva en cómo conocemos la verdad ha resultado en la desestimación de logros cognitivos cuya naturaleza, se argumenta, no puede explicarse plenamente sólo en términos de esa piedra de toque tradicional. Se piensa que uno de esos logros cognitivos desestimados es el entendimiento. ${ }^{1}$

Sea o no que el dominio de la creencia verdadera en la arquitectura de la evaluación epistémica se deba a la concentración en el escepticismo, el hecho es que ese dominio ha sido real y es identificable en el teorizar de muchos epistemólogos contemporáneos. En este trabajo, y tomando prestada terminología introducida por Goldman (1999, cap. 3; y 2001), llamaré "veritista" a un tipo de teoría de la evaluación epistémica que considera que el valor de la creencia verdadera está esencialmente involucrado en la explicación de todo valor epistémico. ${ }^{2}$ La epistemología veritista no niega que exista una pluralidad de valores epistémicos: el conocimiento y la justificación ofrecen ya un par de ejemplos de bienes epistémicos distintos de la creencia verdadera; lo que sostiene es sólo que la explicación del valor epistémico de esos bienes necesariamente tiene que aludir al valor de la creencia verdadera.

La tesis de que la explicación de todo valor epistémico esencialmente debe hacer referencia al valor de la creencia verdadera es indeterminada: no dice de qué manera exactamente debe hacerse referencia a tal valor. Deliberadamente doy esta caracterización amplia del veritismo con el fin de que abarque distintintas teorías veritistas específicas.

Algunas teorías veritistas que llamo "duras" consideran que la creencia verdadera es el único valor epistémico fundamental; dentro de una teoría semejante, la razón específica por la cual tiene que hacerse refe-

${ }^{1}$ Kvanvig 2003 y Zagzebski 2001 señalan que se debe a la concentración teórica en el escepticismo el que se hayan desestimado logros epistémicos, como el entendimiento, que ellos no consideran susceptibles de tratamiento adecuado en el marco de una epistemología que tenga como piedra de toque la creencia verdadera. Riggs menciona la sabiduría como otro de esos logros cognitivos ignorados; de hecho, piensa que la sabiduría es el "bien epistémico más alto", del cual el entendimiento es meramente un componente (Riggs 2003, p. 216).

${ }^{2}$ Debo señalar que Goldman no caracteriza exactamente de esta manera la epistemología veritista. Cuál sea la mejor caracterización de la tesis central del veritismo es una cuestión compleja que no puedo discutir en este trabajo. Aquí simplemente formulo lo que me parece ser el compromiso más neutral y básico de las epistemologías veritistas.

Diánoia, vol. LV, no. 65 (Noviembre 2010). 
rencia al valor de la creencia verdadera en la explicación de cualquier valor epistémico es que el valor epistémico de cualquier unidad que se esté evaluando (un método, una propiedad, un rasgo de carácter, etc.) es meramente instrumental con respecto al logro de la creencia verdadera. ${ }^{3}$ De este modo, la evaluación distintivamente epistémica de una unidad se realiza desde el punto de vista de qué tan conducente sea al logro del valor epistémico fundamental que es la creencia verdadera.

Pero no todas las teorías veritistas son duras; es decir, no todas suponen que la manera en la que el valor de la creencia verdadera está involucrado en la explicación de todo valor epistémico sea en calidad de único valor epistémico fundamental con respecto al cual cualquier otro valor epistémico sea meramente instrumental. Hay teorías veritistas, que llamaré "tolerantes", que permiten que el papel del valor de la creencia verdadera en la explicación de algunos valores epistémicos sea distinto del papel monista que le atribuyen las teorías duras. Por ejemplo, según un tipo de teoría defendida por Sosa (2003 y 2007, cap. 4) y Greco (2003 y 2010), existe un tipo de valor epistémico que consiste en el mérito de un sujeto al lograr una creencia verdadera mediante el ejercicio de alguna competencia cognitiva propia. De acuerdo con este tipo de teoría, el valor epistémico de ese mérito no es meramente instrumental con respecto al logro de la creencia verdadera, pues un sujeto puede adquirir el mismo número de creencias verdaderas sin mérito alguno; por ejemplo, sólo gracias a la ayuda externa de un ángel guardián o de un genio benigno. De modo que el valor epistémico contenido en el mérito de lograr la verdad mediante las competencias propias no es explicable simplemente por el hecho de que tales competencias sean conducentes a la creencia verdadera. Sin embargo, la explicación del valor del mérito epistémico todavía hace referencia de algún otro modo al valor de la creencia verdadera; en particular, lo que hace que el valor de ese mérito sea distintivamente epistémico es que el bien que se le acredita al sujeto es el bien epistémico de la creencia verdadera. Si el bien que se le acreditara al sujeto en virtud del ejercicio de sus competencias no fuera la creencia verdadera sino un bien estético, moral o atlético, entonces el mérito del sujeto no poseería un valor distintivamente epistémico sino estético, moral o deportivo. De este modo, el valor de la creencia verdadera sigue desempeñando un papel esencial en la explicación del valor distintivo del mérito epistémico.

${ }^{3}$ Véase una discusión de este tipo de teoría veritista en Pritchard 2010, secciones 1.4 y 1.5; Pritchard la llama "Monismo-V del valor epistémico".

Diánoia, vol. LV, no. 65 (Noviembre 2010). 
Este tipo de teorías sobre el valor del mérito epistémico son sólo un ejemplo de cómo el valor de la creencia verdadera puede estar esencialmente involucrado en la explicación del valor epistémico de algo, sin asumir que todo valor epistémico sea meramente instrumental con respecto al valor de la creencia verdadera. Puede haber otras maneras distintas en las que el valor de la creencia verdadera puede estar involucrado en la explicación de otros valores epistémicos, pero para los fines de este trabajo es suficiente considerar como un ejemplo la explicación del valor del mérito epistémico.

Debo subrayar que las epistemologías veritistas tolerantes, tal como las he caracterizado, no niegan que algunos valores epistémicos sean correctamente explicables como meramente instrumentales con respecto al valor de la creencia verdadera; lo que rechazan es la tesis dura de que todos los valores epistémicos sean correctamente explicables de esa manera. Al permitir que el valor de la creencia verdadera desempeñe más de un único papel en la explicación de diversos valores epistémicos, las epistemologías veritistas tolerantes poseen, en principio, un poder explicativo superior al de las teorías veritistas duras.

En este trabajo discutiré una serie de objeciones a las epistemologías veritistas según las cuales éstas no consiguen explicar satisfactoriamente la naturaleza y el valor epistémico de un tipo central de entendimiento que llamaré entendimiento temático, es decir, el entendimiento que puede lograrse de un cuerpo complejo de información sobre un tema, asunto $\mathrm{u}$ objeto. Asumo que el entendimiento temático es un tipo de logro cognitivo que una epistemología debe ser capaz de explicar. Argumentaré que las objeciones sometidas a examen no consiguen revelar ningún déficit explicativo en las epistemologías veritistas tolerantes, tal como las caractericé antes. Este tipo de teoría veritista es suficientemente robusta para dar cuenta de todo lo que una epistemología tendría que poder explicar acerca de la naturaleza y el valor epistémico del entendimiento. Si la epistemología veritista sufre de deficiencias, éstas deben buscarse en otra parte, no en los déficits explicativos que le imputan las objeciones que examinaré.

Las secciones 2 y 3 se ocupan de objeciones que intentan mostrar que los estados cognitivos implicados en el entendimiento tienen una naturaleza sui generis que no es proposicional y que, por lo tanto, el modelo veritista de evaluación no puede aplicárseles por estar centrado en la evaluación de la creencia verdadera, que es necesariamente proposicional. Las secciones 4 y 5 examinan objeciones que intentan mostrar que hay aspectos del entendimiento epistémicamente valiosos que no pueden explicarse en términos veritistas.

Diánoia, vol. LV, no. 65 (Noviembre 2010). 
Antes de continuar debo hacer un par de observaciones. Primero, las objeciones al veritismo que discutiré no se presentan de manera aislada en los textos de los autores que las formulan (principalmente J. Kvanvig, L. Zagzebski y W. Riggs). ${ }^{4}$ De hecho, el propósito central de esos textos no es socavar el veritismo, sino presentar una teoría positiva sobre la naturaleza y el valor del entendimiento que no tenga las supuestas deficiencias de una teoría veritista. En este trabajo no me ocupo de las teorías positivas sobre el entendimiento propuestas por esos autores, sino sólo de las objeciones que hacen al veritismo; ésta es una estrategia legítima, pues si sus objeciones al veritismo fracasan, las teorías positivas que ellos proponen perderán una de sus motivaciones centrales. Segundo, los términos que uso para describir las objeciones de esos autores no siempre coincide con la terminología que ellos mismos usan. Esto podría dar la impresión de que las objeciones que les atribuyo realmente no se encuentran en los textos, pero esto no es así. Ofrezco suficientes citas que muestran cómo el espíritu de las reflexiones de esos autores es antiveritista en el sentido que yo sostengo. Si a veces uso terminología que ellos no utilizan para describir lo que desean objetar del veritismo es simplemente con el fin de acuñar una manera uniforme de referirme a esas objeciones.

\section{Entender, saber y estados dóxicos}

Como ya he señalado, el tipo de entendimiento del que me ocuparé es el entendimiento que uno puede tener de un cuerpo complejo e integrado de información; se trata del entendimiento o comprensión que uno puede lograr con respecto a un tema o materia. Por ejemplo, entender un determinado periodo en la historia de una nación o el funcionamiento de un artefacto. En lo que sigue se aclarará más en qué consiste este tipo de entendimiento; parte de lo que está en juego en las objeciones antiveritistas que examinaré es precisamente determinar en qué consiste exactamente el entendimiento temático.

Se ha argumentado que el entendimiento temático no es un tipo de conocimiento proposicional. J. Kvanvig, por ejemplo, cree que "el entendimiento es un logro cognitivo distinto del conocimiento", y argumenta en contra de la concepción según la cual "la clase de entendimiento en cuestión cuando consideramos nuestros éxitos y logros cognitivos es algún tipo de conocimiento profundo y comprensivo acerca

${ }^{4}$ En la bibliografía puede verse la referencia completa de los textos de estos autores a los que me refiero.

Diánoia, vol. LV, no. 65 (Noviembre 2010). 
de algún tema, tópico o cuestión particular" (Kvanvig 2003, p. 188). ${ }^{5}$ El entendimiento al que se refiere Kvanvig consiste en "una aprehensión o apreciación interna de cómo los variados elementos en un cuerpo de información se relacionan entre sí en términos de relaciones explicativas, lógicas, probabilísticas y de otro tipo" (2003, pp. 192-193). La tesis en favor de la que argumenta es que el entendimiento, en ese sentido, no es una especie de conocimiento. Su argumento principal en favor de esta tesis consiste en mostrar que algo que es esencial para el conocimiento - a saber, que la creencia no sea accidentalmente verdaderano es esencial para el entendimiento; por lo tanto, el entendimiento no puede ser una especie de conocimiento:

el entendimiento no refiere a los aspectos etiológicos que pueden ser cruciales para el conocimiento. Lo que es distintivo del entendimiento [...] es el ver o apreciar interno de relaciones explicativas e inductoras de coherencia en un cuerpo de información [... ]. Cuando pensamos sobre el conocimiento [...] nuestra atención se dirige inmediatamente hacia otra parte [...], tomamos en cuenta la posibilidad de que acertemos de manera fortuita, por accidente, de estar en lo correcto pero solamente por suerte. (2003, p. 198)

Kvanvig describe un caso en el que un sujeto tiene entendimiento de un cuerpo de información sin tener conocimiento de las proposiciones que integran ese cuerpo, precisamente porque el sujeto es beneficiario de una clase de suerte epistémica incompatible con el conocimiento. Juan ha leído mucho sobre la historia del dominio comanche de las planicies meridionales de Norteamérica durante el siglo XIX. Los libros que Juan leyó contienen información correcta sobre este asunto, de modo que al leerlos Juan ha obtenido un entendimiento impresionante de ese fenómeno histórico. Ahora bien, supóngase que la mayoría de los libros sobre ese tema que Juan no consultó contienen información incorrecta sobre ese asunto. Ocurre que Juan ha consultado los libros de historia que contienen información correcta, pero su elección de libros para consulta ha sido aleatoria: fácilmente podría haber consultado los libros que contienen información incorrecta, simplemente tuvo suerte en no consultar esos libros. Kvanvig sostiene que, debido a que Juan posee información correcta sobre los hechos históricos como resultado de esa suerte, Juan no tiene conocimiento de esos hechos. ${ }^{6}$

${ }^{5}$ Las traducciones de todas las citas de textos originales en inglés son mías.

${ }^{6}$ Se asume que el tipo de suerte en cuestión aquí es el tipo de suerte que impide el conocimiento en un caso de granero falso [fake barn] que originalmente describió Goldman 1976.

Diánoia, vol. LV, no. 65 (Noviembre 2010). 
Pero Kvanvig alega que, a pesar de que Juan no tenga conocimiento de las proposiciones que componen su cuerpo de información acerca del dominio comanche, tiene entendimiento de ese fenómeno histórico. El entendimiento sobrevive a una clase de suerte epistémica que es fatal para el conocimiento; por lo tanto, el entendimiento no puede ser una especie de conocimiento.

La tesis de que, en un caso como el anterior, la clase de suerte que se ilustra impide el conocimiento pero no el entendimiento puede cuestionarse; sin embargo, en lo que sigue concederé esa tesis y que de ella se sigue que el entendimiento temático no es una especie de conocimiento. ${ }^{7}$ Argumentaré que aun cuando esto sea correcto, explicar la naturaleza del entendimiento no constituye un problema insuperable para un modelo veritista de los logros epistémicos.

En primer lugar, intentemos entender mejor qué es exactamente el entendimiento temático, tal como Kvanvig lo describe. En la cita anterior, Kvanvig afirma que el entendimiento "requiere [...] una aprehensión [grasping] o apreciación [appreciation] interna de cómo los diversos elementos en un cuerpo de información se relacionan entre sí en términos de relaciones explicativas, lógicas, probabilísticas y de otros tipos" (2003, pp. 192-193), se trata de "el ver [seeing] o apreciar interno de relaciones explicativas e inductoras de coherencia en un cuerpo de información" (2003, p. 198). De acuerdo con él, alguien logra entender cuando "la persona ha visto [seen] los tipos correctos de relaciones entre las diversas piezas de información que aprehende" (2003, p. 193), y pone énfasis en que el mero hecho de que se den esas relaciones entre las piezas de información que el sujeto posee no es suficiente para que se dé el entendimiento; éste requiere, además, que "la manera en que toda

${ }^{7}$ Grimm 2006 argumenta en detalle que Kvanvig se equivoca al pensar que el conocimiento y el entendimiento no van de la mano en casos como el de la historia comanche que describe. Grimm argumenta (pp. 522-530) que, dependiendo de los detalles del caso, si un sujeto no tiene conocimiento tampoco tiene entendimiento, y si tiene conocimiento también tiene entendimiento; como él dice: "cuando el conocimiento y el entendimiento cambian según las circunstancias particulares, cambian juntos" (p. 530). Si el conocimiento y el entendimiento son igualmente vulnerables o igualmente inmunes a los mismos tipos de suerte epistémica, entonces Kvanvig no puede concluir que el entendimiento no es una especie de conocimiento a partir de la premisa de que el conocimiento y el entendimiento son afectados de diferente manera por esos tipos de suerte epistémica, pues esta premisa sería falsa. Sin embargo, en este trabajo deseo conceder la premisa de Kvanvig y su conclusión de que el entendimiento no es una especie de conocimiento; mostraré que, a pesar de estas concesiones, no hay razón convincente para pensar que el entendimiento sea intratable para el veritismo.

Diánoia, vol. LV, no. 65 (Noviembre 2010). 
la información encaja entre sí tiene que ser parte de lo que la persona es consciente [aware of]" (2003, p. 202). ${ }^{8}$ Tenemos que preguntar en qué consiste este distintivo "aprehender", "ver", "apreciar" o "ser consciente de" las relaciones explicativas en el entendimiento. Puesto que en el lenguaje ordinario los usos más naturales de tales expresiones son fácticos (es decir, se usan correctamente sólo cuando la proposición que sigue a la clausula-que que acompaña a esas expresiones es verdadera), podríamos formular la hipótesis de que el significado de tales vocablos es equivalente $\mathrm{a}-\mathrm{o}$ por lo menos implica-conocimiento proposicional, pues éste es el estado mental fáctico más general. ${ }^{9}$ De acuerdo con esto, "apreciar" cómo se integran entre sí los diversos elementos en un cuerpo de información y "ver" las relaciones explicativas que los vinculan implicaría saber que tales relaciones se dan. Sin embargo, Kvanvig no puede asumir esta lectura natural de esos vocablos porque tal lectura haría que el saber que se dan las relaciones explicativas relevantes fuera una condición necesaria para entender el cuerpo de información correspondiente, lo cual significa que uno no podría entender ese cuerpo de información sin tener conocimiento de las relaciones explicativas relevantes que se dan entre los elementos de información. Pero esto parece contradecir su tesis central de que el entendimiento no es una especie de conocimiento porque uno puede entender el cuerpo de información sin tener conocimiento de las proposiciones individuales que lo constituyen.

Kvanvig podría replicar que el conocimiento de las relaciones explicativas entre las proposiciones que componen un cuerpo de información no es lo mismo que el conocimiento de las proposiciones mismas que están relacionadas; podríamos negar que el segundo conocimiento sea necesario para el entendimiento del cuerpo de información (como Kvanvig lo hace con base en su ejemplo sobre la historia comanche) y aceptar consistentemente con ello que el primer conocimiento es necesario para ese entendimiento (como lo demanda la lectura fáctica de sus expresiones clave). Sin embargo, si el ejemplo de Kvanvig muestra que se puede entender un cuerpo de información sin tener conocimiento de las proposiciones individuales que lo constituyen, también muestra que se puede tener entendimiento sin tener conocimiento de las relaciones explicativas relevantes que se dan entre esas proposiciones. En efecto, en el ejemplo de Kvanvig, si la suerte epistémica es fatal para el conocimiento de las proposiciones individuales en el cuerpo de información que uno posee,

${ }^{8}$ Todas las cursivas en las citas de este párrafo son mías.

${ }^{9}$ En el sentido definido por Williamson 2000, p. 39. 
también lo es para el conocimiento de las relaciones explicativas que se dan entre ellas, porque uno aprende acerca de esas relaciones a través de la misma fuente de información, la cual es correcta, pero uno ha tenido suerte al seleccionarla. Así pues, en su ejemplo, Kvanvig tiene que aceptar que la suerte epistémica también impide el conocimiento de las relaciones explicativas que se dan entre los elementos de información que uno posee, sin impedir el entendimiento de ese cuerpo de información. Por lo tanto, Kvanvig no puede aceptar que el entendimiento implique el conocimiento de que tales relaciones se dan. Kvanvig no puede adoptar coherentemente la lectura natural, fáctica, de los términos que usa para caracterizar la naturaleza del entendimiento.

En una lectura menos natural de los términos clave que Kvanvig usa para caracterizar el entendimiento, ellos resultan meramente dóxicos: cuando uno "aprecia" o "aprehende" que $p$, uno juzga —o al menos está dispuesto a juzgar - que $p$. Esta lectura hace de los estados de apreciar y aprehender que $p$ especies de creer que $p$; en lo que sigue entenderé "estados dóxicos" como estados de creencia. Puesto que toda creencia es proposicional, según la presente lectura, el entendimiento consistiría en creer ciertos contenidos proposicionales complejos que articulan las relaciones explicativas establecidas entre las diversas piezas de información que uno posee. Dada nuestra discusión previa, hemos de conceder que tales creencias complejas no necesitan ser conocimiento para constituir entendimiento. No obstante, es claro que no cualquier conjunto de tales creencias complejas lo dotaría a uno de entendimiento. Si uno forma creencias de manera extremadamente descuidada que, sin embargo, articulan correctamente relaciones explicativas entre varios hechos que uno ha memorizado sobre la historia comanche, no por ello uno entiende algo sobre la historia comanche. La razón de esta carencia de entendimiento no es que la manera en la que uno llegó a esas creencias no sea fiable, que fácilmente podría haber conducido a la falsedad o que entregue creencias verdaderas sólo por accidente. Todos estos son obstáculos para el conocimiento de esas proposiciones en el caso presente, pero hemos visto que, en la concepción de Kvanvig, podemos carecer de ese conocimiento sin carecer del entendimiento correspondiente. La razón por la que uno carece de entendimiento en el caso que imaginamos debe ser diferente. Propongo que la razón es que creemos las proposiciones que articulan las relaciones explicativas a pesar de tener razón para dudar de la fiabilidad de la fuente de esas creencias, pues sabemos que formar creencias de manera extremadamente descuidada sobre cómo se relacionan entre sí varios hechos que hemos memorizado no es un buen camino para llegar a entender cómo 
se relacionan esos hechos entre sí. En un caso tal, creemos en varias relaciones explicativas por malas razones, no conseguimos creer con justificación adecuada.

Lo anterior sugiere que las creencias que articulan las relaciones explicativas entre diversos elementos de información tienen que estar adecuadamente justificadas si es que han de dar lugar al entendimiento. Pero, entonces, el entendimiento se convierte en cúmulos de una especie de creencia verdadera justificada y así el veritismo puede explicar su naturaleza como un caso especial de creencia verdadera justificada. ${ }^{10}$ En este punto, desde luego, podría haber explicaciones no veritistas del componente de justificación de ese caso especial de creencia verdadera justificada, tal como puede haber explicaciones no veritistas de la justificación en general. Por ejemplo, un pragmatista desearía explicar la justificación no sólo en términos de alguna conexión adecuada con la verdad, sino además, y tal vez principalmente, en términos de alguna conexión adecuada con los fines prácticos de las personas. Sin embargo, el asunto que importa es que el veritismo podría dar una explicación de los estados dóxicos que, según la presente hipótesis, constituyen los estados de entendimiento. La naturaleza del entendimiento no sería intratable para una epistemología veritista.

La lectura dóxica de los términos clave de Kvanvig ayudaría de la manera indicada a reivindicar el veritismo en contra del cargo de que no es capaz de explicar la naturaleza del entendimiento temático. Probablemente ésta sea la razón de que Kvanvig desee evitar que se interpreten los estados de aprehensión y apreciación de relaciones explicativas como estados dóxicos. La base de su rechazo de la lectura dóxica es que:

cuando se logra el entendimiento, el objeto del entendimiento es un "trozo informacional" más que un número de proposiciones singulares [...]. Aunque uno podría saber un cierto número de proposiciones, incluso junto con otras proposiciones acerca de las relaciones explicativas y de otro tipo inductoras de coherencia entre la información en cuestión, este conocimiento proposicional no constituiría entendimiento [...] hasta que ocurriera un cambio en el objeto de tal logro cognitivo. (2003, p. 192)

Puesto que el objeto de la creencia es proposicional, tanto como lo es el objeto del conocimiento proposicional, estas observaciones de Kvanvig

\footnotetext{
${ }^{10}$ Lo cual es consistente con la insistencia de Kvanvig en que el entendimiento no es una especie de conocimiento, pues, tal como se acepta en la era pos-Gettier, la creencia verdadera justificada no es suficiente para el conocimiento.
}

Diánoia, vol. LV, no. 65 (Noviembre 2010). 
implican que el apreciar relaciones explicativas constitutivo del entendimiento no puede ser una especie de creencia; el entendimiento es otro tipo de estado sui generis cuyo objeto no es proposicional, sino un "trozo informacional". Pero entonces Kvanvig nos deja por completo en la oscuridad en lo que se refiere a cómo interpretar los estados de apreciación implicados en el entendimiento. En vista de su meta argumentativa (i.e., defender que el entendimiento no es una especie de conocimiento), no puede adoptar de manera coherente la interpretación natural, fáctica, de los estados de apreciación de relaciones explicativas; pero tampoco desea adoptar la lectura dóxica, la cual haría tratable el problema de la naturaleza del entendimiento dentro de un enfoque veritista. Kvanvig no ofrece una caracterización clara de los supuestamente sui generis estados cognitivos implicados en el entendimiento que, según piensa, lo hacen problemático para un enfoque veritista. $^{11}$

\section{Vehículos no proposicionales de entendimiento}

En la sección anterior sugerí que si interpretamos los estados de apreciación de relaciones explicativas implicados en el entendimiento como estados dóxicos, el veritismo podría tratarlos como una especie de creencias verdaderas justificadas. Algunos oponentes del veritismo siguen a Kvanvig en su rechazo a este intento de hacer el entendimiento tratable para el veritismo argumentando que los estados de entendimiento no son necesariamente proposicionales y que, por lo tanto, a fortiori, no pueden explicarse como una especie de creencias verdaderas justificadas, pues toda creencia es necesariamente proposicional. L. Zagzebski, por ejemplo, cree que "entender involucra ver cómo las

${ }^{11}$ La concepción positiva de Kvanvig sobre la naturaleza del entendimiento se vuelve confusa dada su afirmación final de que "el entendimiento [... ] está constituido por creencias verdaderas subjetivamente justificadas a lo largo de un cuerpo de información, circunscrito de manera apropiada, que se sistematiza y organiza en el proceso en el que se logra el entendimiento" (2003, p. 202; las cursivas son mías). Con el fin de ser coherente, Kvanvig no debe querer decir que los estados de apreciación de relaciones explicativas entre elementos de información, que son distintivos del entendimiento, sean ellos mismos "creencias verdaderas subjetivamente justificadas", pues entonces el objeto de tales estados sería proposicional, contrariamente a lo que hemos visto que desea sostener. Lo que debe querer decir es que los elementos de información entre los que uno aprecia ciertas relaciones explicativas cuando uno entiende, son "creencias verdaderas subjetivamente justificadas", dejando aún sin explicar en qué consiste la apreciación o aprehensión misma de esas relaciones explicativas.

Diánoia, vol. LV, no. 65 (Noviembre 2010). 
partes de un cuerpo de conocimiento encajan entre sí, donde el encajar entre sí no tiene él mismo forma proposicional" (2001, p. 244, las cursivas son mías). Y en el mismo espíritu, W. Riggs afirma que:

hay buenas razones para dudar que el entendimiento siempre sea proposicional. Esto es, el contenido real del entendimiento que uno tiene podría no ser plenamente explicable en términos de creencias que puedan atribuirse de manera plausible al agente. Esto equivale a negar que el fenómeno del entendimiento pueda reducirse simplemente a una colección de creencias verdaderas. (Riggs 2003, p. 218)

Como Kvanvig, el tipo de entendimiento que Zagzebski y Riggs tienen en mente involucra la apreciación de un patrón de relaciones explicativas y de otro tipo que se mantienen dentro de un todo. Tal como Riggs lo indica:

La clase de entendimiento que tengo en mente es la apreciación o aprehensión de un orden o patrón y de cómo las cosas se relacionan entre sí. El entendimiento tiene una multitud de objetos apropiados, entre ellos máquinas complejas, personas, materias o disciplinas, pruebas matemáticas y muchos más. (Riggs 2003, p. 217)

Ni Zagzebski ni Riggs niegan que entender un tema o una máquina involucre tener varios estados proposicionales; por ejemplo, aquellas creencias que uno expresaría verbalmente cuando articula lo que entiende. Lo que niegan es que el entendimiento consista en o sea plenamente explicable en términos del hecho de tener esos estados proposicionales. Con el fin de justificar esta afirmación discuten casos en los que la apreciación o aprehensión de relaciones en un cuerpo de información se obtiene a través de un vehículo no proposicional, que no sería plausible reducir a un conjunto de proposiciones creídas por la persona que obtiene el entendimiento. Riggs menciona el entendimiento que se obtiene a través de gráficas y diagramas (2003, p. 218) y Zagzebski menciona el papel de una imagen metafórica:

El genetista matemático, Sewall Wright, usó la metáfora de una planicie con valles y colinas como una manera de explicar los mecanismos genéticos de adaptación. Argumentó que la selección natural lleva a las poblaciones cuesta arriba. Científicos que piensan que las poblaciones buscan el nivel cercano más bajo han sugerido invertir la imagen. De cualquier manera, la gente encuentra que la imagen les ofrece un entendimiento que de otra forma no habrían logrado. (Zagzebski 2001, pp. 241-242) 
Vimos que Kvanvig sostiene que la razón por la que el entendimiento no puede reducirse a la creencia es que el objeto del entendimiento no es proposicional, sino más bien un "trozo informacional", pero señalé que no consigue dar una explicación inteligible de qué pueda ser esto si no es un conjunto de proposiciones. Zagzebski y Riggs ofrecen una hipótesis más clara de por qué esa reducción no es posible. La idea es que el entendimiento que se obtiene a través de ciertos vehículos (tales como un diagrama, una gráfica o una imagen metafórica) no se obtendría si el "trozo informacional" relevante fuera representado (si fuera posible hacerlo) por otros medios. Ahora bien, esos vehículos mediante los que uno obtiene el entendimiento en cuestión no tienen estructura proposicional y, por esto, ellos piensan que el entendimiento obtenido a través de esos vehículos no puede consistir en -o ser explicable en términos de-creencias verdaderas que podamos atribuir al sujeto, pues las creencias necesariamente tienen contenidos proposicionales. En su concepción, los estados de entendimiento y los estados de creencia resultan ser estados cognitivos con tipos de contenido muy diferentes.

Una respuesta inicial a esta objeción sería que, en el mejor de los casos, sólo presenta un problema local para el veritismo, pues no todos los casos de entendimiento se logran a través de vehículos que no tienen estructura proposicional. Si lo que hace problemáticos los casos de entendimiento que Zagzebski y Riggs consideran es que se logran mediante el uso de vehículos no proposicionales, entonces todos aquellos casos de entendimiento que sí se logran mediante vehículos proposicionales (por ejemplo, leyendo o escuchando oraciones significativas) podrían ser tratados en un marco veritista como algún tipo de creencia. Sin embargo, puede cuestionarse que aun los casos de entendimiento que se logran mediante vehículos no proposicionales sean problemáticos para el veritismo. Debemos notar que una presuposición del razonamiento de Zagzebski y Riggs parece ser la idea de que si el entendimiento se logra a través de un vehículo que no tiene estructura proposicional, como una gráfica, un diagrama o una imagen metafórica, entonces el estado de entendimiento mismo no tiene contenido proposicional. Pero esta suposición necesita defensa, pues sin duda puede ponerse en duda. Quizá el vehículo usado para obtener entendimiento en una ocasión particular no tenga estructura proposicional, pero tal vez el estado que constituye el que uno entienda sí tenga contenido proposicional. Por ejemplo, una gráfica puede ser un aditamento excelente para entender alguna tendencia central en los datos recabados hasta el momento en relación con cierto fenómeno social. La representación visual de la tendencia es sin 
duda un valioso auxiliar para el logro del entendimiento, y ese auxiliar puede ser difícil de reemplazar con medios alternativos de representación que tengan los mismos efectos o ventajas. Pero estos hechos se refieren a la conveniencia práctica de utilizar ciertos medios específicos de representación con el fin de lograr entender algo, no implican por sí solos que el entendimiento logrado no consista en un estado mental que tenga contenido proposicional. Después de todo, uno puede expresar el entendimiento que ha conseguido del fenómeno en cuestión diciendo que los datos recabados muestran tal-y-cual tendencia, y que esto sugiere que tal-y-cual es el caso; y estas proferencias evidentemente comunican estados proposicionales, de ahí que su formulación contenga cláusulas-que explícitas.

Las ventajas prácticas de utilizar un vehículo no proposicional pueden manifestarse no sólo en la adquisición del entendimiento, sino también en su transmisión. Puede ser que sea más conveniente utilizar el vehículo original que no tiene estructura proposicional en lugar de algún otro, particularmente en lugar del discurso oral sin auxilios visuales, con el fin de representar y transmitir el entendimiento que uno ha logrado. Pero, de nuevo, esto no prueba que el estado de compresión que se ha logrado no tenga contenido proposicional; únicamente indica que el vehículo original facilita el logro de ciertos propósitos relevantes de comunicación. Expresar de manera verbal el entendimiento que uno ha logrado, en comparación con dibujar una gráfica sobre el pizarrón, puede ser impráctico, dadas ciertas metas pedagógicas, o pragmáticas de otro tipo, porque puede no ser la mejor manera de resaltar alguna tendencia central que se manifiesta en los datos recabados y que es crucial para entender el fenómeno en cuestión. No obstante, la tendencia central manifestada en los datos puede ser representada y expresada proposicionalmente.

En cualquier caso, la cuestión importante es que Zagzebski y Riggs necesitan argumentar en favor de la tesis de que en los casos de entendimiento que ellos consideran, no sólo el vehículo para conseguir, representar y transmitir el entendimiento no tiene estructura proposicional, sino que el estado mismo de entendimiento, logrado a través de ese vehículo, no tiene contenido proposicional. Ellos no muestran que esto sea así y he dado razones para pensar que de hecho no es así. ${ }^{12}$

${ }^{12}$ Zagzebski y Riggs podrían responder retando al veritista a que en un caso específico de entendimiento - por ejemplo, el entendimiento sobre cómo funciona una máquina, que se logra mediante la inspección de un diagrama- dé expresión completa de lo que entiende en términos de creencias verdaderas obtenidas

Diánoia, vol. LV, no. 65 (Noviembre 2010). 
No hemos encontrado hasta aquí ninguna razón convincente para pensar que la naturaleza de los estados cognitivos implicados en el entendimiento temático, o el papel que desempeñan los vehículos a través de los cuales uno puede lograr entender algo, sean problemáticos para un enfoque veritista de evaluación epistémica.

\section{El valor epistémico de la sistematicidad y la organización}

Los oponentes del veritismo se interesan en plantear objeciones que se enfocan ya no en la naturaleza actitudinal de los estados cognitivos implicados en el entendimiento, ni en cuál sea su tipo de contenido propio, sino en el valor epistémico de tales estados; argumentan que el veritismo no puede dar cuenta de algunos aspectos en los que el entendimiento es distintivamente valioso. Kvanvig escribe:

dominar tales relaciones explicativas [constitutivas del entendimiento] no es valioso sólo porque involucre descubrir verdades nuevas, sino también porque descubrir tales relaciones organiza y sistematiza nuestro pensamiento acerca de un tema de una manera que va más allá de la mera adición de más creencias verdaderas, o incluso de más creencias verdaderas justificadas. (2003, p. 202; las cursivas son mías)

Esto es correcto, sin duda. El valor de entender un tema va más allá del valor de meramente tener un número creciente de creencias verdaderas acerca de ese tema; desde el punto de vista de Kvanvig, el valor excedente en cuestión consiste en algún tipo de organización y sistematización. Podemos conceder que esto es así, pero tenemos que preguntar si la naturaleza de ese valor es epistémica. Parte del valor asociado a las virtudes de tener un pensamiento organizado y sistemático sobre algún tema es sin duda alguna epistémico. Por ejemplo, la organización y sistematicidad propias del entendimiento de un cuerpo de información son conducentes a metas cognitivas que claramente tienen

a través de la inspección del diagrama. La cuestión de si un sujeto puede o no dar expresión proposicional completa de lo que entiende en un caso como éste es una cuestión empírica que no puedo responder aquí. En cualquier caso, no tengo que contestar esta pregunta, pues mi objetivo es simplemente argumentar que del hecho (correctamente identificado por Zagzebski y Riggs) de que el entendimiento pueda lograrse mediante vehículos no proposicionales no se sigue que el estado mismo de entendimiento no tenga estructura proposicional. La cuestión de si un sujeto puede articular y dar expresión a todos los estados mentales proposicionales que constituyen su entendimiento en un caso particular es la cuestión empírica que no es necesario contestar aquí.

Diánoia, vol. LV, no. 65 (Noviembre 2010). 
un valor epistémico explicable en términos veritistas; dos de esas metas son la predicción y la explicación. En efecto, una predicción y una explicación son epistémicamente valiosas al menos porque la probabilidad de que sean verdaderas es alta, dadas las evidencias. De manera que en la medida en que las virtudes de la organización y la sistematicidad sean conducentes a obtener predicciones y explicaciones cuya probabilidad de ser verdaderas sea alta, en esa medida poseerán ellas mismas valor epistémico. De este modo, el valor epistémico de esas virtudes será explicado en términos veritistas en función de su capacidad de conducir a explicaciones y predicciones cuya probabilidad de resultar verdaderas sea alta, dadas las evidencias.

Pero la objeción de Kvanvig es precisamente que la organización y sistematización propias del entendimiento son valiosas por algo más que simplemente el hecho de que hagan más probable la adquisición de nuevas verdades. Desde el punto de vista de Kvanvig, señalar que la organización y sistematización son epistémicamente valiosas porque son conducentes a buenas predicciones y explicaciones, y que éstas, a su vez, son epistémicamente valiosas en la medida en que su probabilidad de ser verdaderas sea alta dadas las evidencias, simplemente mueve el problema de lugar sin resolverlo. En efecto, Kvanvig podría repetir su objeción ahora con respecto a la predicción y la explicación, alegando que estas metas no son epistémicamente valiosas sólo porque tengan una probabilidad alta de ser verdaderas dadas las evidencias. Me parece que en esta coyuntura la carga de la prueba está del lado del antiveritista: su oponente, el veritista, ha identificado un aspecto en el que una predicción o una explicación son epistémicamente valiosas, el cual puede explicarse en el marco de su modelo de evaluación. Si el antiveritista considera que existen otros aspectos de esas metas cognitivas que son epistémicamente valiosas, pero inexplicables en términos veritistas, tiene que identificarlos y mostrar que el veritismo, o cualquier teoría de la evaluación epistémica, tendría que dar cuenta de su valor. Pero, de hecho, los antiveritistas citan típicamente como aspectos de logros cognitivos que supuestamente son intratables para el veritismo aspectos tales que no hay razón para pensar que una teoría adecuada de la evaluación epistémica tuviera que poder dar cuenta de ellos. Por ejemplo, al explicar por qué el valor de las virtudes inicialmente citadas de la organización y la sistematicidad son problemáticas para el veritismo, Kvanvig menciona los siguientes aspectos de esas virtudes como aspectos valiosos, pero cuyo valor es inexplicable en términos veritistas: 
Tal organización es pragmáticamente útil porque nos permite razonar a partir de una parte de la información hacia otra información relacionada que es útil como una base para la acción [...]. Asimismo, tales elementos organizados en nuestro pensamiento proveen un punto final al proceso de investigación el cual es intrínsecamente satisfactorio, y produce así una sensación o sentido de completud en nuestra aprehensión de un tema particular. (Kvanvig 2003, p. 202; las cursivas son mías.)

Es verdad que entender un tema es valioso por esas razones: por ser pragmáticamente útil y por producir una sensación satisfactoria de completud y logro en la comprensión que tenemos de las cosas; pero no se nos ha dado una razón para conceptualizar esos valores como epistémicos. La práctica evaluativa estándar se encuentra equipada con taxonomías no epistémicas que clasifican esos tipos de valor como, por ejemplo, prácticamente útil y psicológicamente agradable. Aunque la aplicación correcta de estas categorías no excluye la presencia de algún valor epistémico, tampoco la implica. El hecho de que una explicación sea prácticamente útil o psicológicamente agradable no excluye que también sea epistémicamente valiosa; sin embargo, puede ser prácticamente útil y psicológicamente agradable sin tener ningún mérito epistémico, por ejemplo, porque se apoya en hipótesis erradas que sólo por accidente parecen explicar el fenómeno particular del momento, pero que fallarían en cualquier otro caso. Las dimensiones de valor que Kvanvig asocia con la organización y la sistematicidad propias del entendimiento son reales, pero no indican necesariamente la presencia de algún valor reconociblemente epistémico que el veritismo tenga que explicar, pues el veritismo es una teoría del valor epistémico de la cognición, no una teoría de todas las posibles clases de valor que la cognición puede traer consigo.

\section{El valor epistémico de habilidades cognitivas}

Otro aspecto del entendimiento que lo hace epistémicamente valioso y que se piensa que es problemático para el veritismo es el hecho de que el entendimiento se encuentra constitutivamente asociado a un rango de habilidades cognitivas. Se argumenta que el valor epistémico de tales habilidades es problemático para el veritismo porque la posesión de tales habilidades no puede explicarse simplemente atribuyendo creencias verdaderas $\mathrm{u}$ otro tipo de estados proposicionales al agente. El sujeto puede tener muchas creencias verdaderas -incluso abundante conocimiento proposicional - acerca del tema sobre el que tiene entendimiento, pero se argumenta que su entendimiento no es reducible al hecho de 
que tenga esas creencias verdaderas o ese conocimiento proposicional, pues la posesión de las habilidades constitutivamente asociadas con el entendimiento no es equivalente a ningún conjunto de esas creencias o esos conocimientos. ${ }^{13}$ Veamos algunos ejemplos que ilustran esta nueva objeción. Riggs nos pide que consideremos a alguien que entiende los automóviles; piensa que el entendimiento de esta persona se "captura" de mejor manera en términos de sus habilidades para predecir y diagnosticar problemas en un automóvil a partir de pistas escasas. Considérese también a alguien que entiende cierto periodo de la historia de una nación; esta persona desplegará su entendimiento en la forma de habilidades para imaginar y discutir competentemente qué habría sucedido en cierta coyuntura histórica si ciertas otras cosas hubiesen sido ligeramente diferentes en el devenir de los hechos. Riggs piensa que esta habilidad de pensar contrafácticamente de manera competente, así como las habilidades de predicción y diagnóstico del experto en automóviles, van más allá de meramente tener creencias verdaderas o conocimiento proposicional acerca de esos temas: ${ }^{14}$ "este tipo de habilidades indican un alto grado de entendimiento, y con frecuencia no pueden adquirirse o explicarse en términos de conocimiento proposicional" (Riggs 2003, p. 220). La idea detrás de este razonamiento parece ser que el entendimiento involucra algún tipo de saber cómo y que éste no puede reducirse al conocimiento proposicional, ni a ningún otro estado cognitivo proposicional del sujeto. Zagzebski es más explícita sobre este punto:

uno no entiende una parte de un área temática si no posee la habilidad de explicar su lugar dentro de una estructura teórica mucho más amplia, y se adquiere la habilidad [ability] para hacer esto cuando se domina una destreza [skill] [...]; el entendimiento es un estado que se consigue mediante el aprendizaje de un arte o una destreza, una technè. Uno consigue entender cuando sabe cómo hacer algo bien, y esto hace que sea una persona fiable para consultarla en asuntos que se refieren a la destreza en cuestión. (2001, p. 241)

${ }^{13}$ La terminología de "asociación constitutiva", "equivalencia" y "reducibilidad" no es la terminología explícita de Riggs ni de Zagzebski, pero considero que puede utilizarse para capturar el espíritu de su objeción.

${ }^{14}$ Woodward (2003) también piensa que el entendimiento debe explicarse en términos de la posesión de ciertas habilidades, en particular habilidades para responder preguntas de la forma "¿Qué habría pasado si las cosas hubiesen sido diferentes?" ["what-if-things-had-been-different?" questions]; en la terminología que usaré, éstas son habilidades para pensar contrafácticamente de manera competente.

Diánoia, vol. LV, no. 65 (Noviembre 2010). 
Zagzebski no niega que saber cómo hacer algo traiga consigo conocimiento proposicional; al contrario, acepta que típicamente es así, por ello afirma que cuando uno sabe hacer algo bien se convierte en un informante fiable sobre asuntos relacionados con la destreza del caso. La cuestión es más bien que tener ese conocimiento proposicional no basta por sí solo para hacer que uno tenga la habilidad. La no reducibilidad del saber cómo al saber proposicional es un asunto debatido, pero por mor del argumento deseo conceder que la tesis no reductivista que Riggs y Zagzebski asumen es correcta; argumentaré que aun así explicar el valor epistémico de las habilidades asociadas con el entendimiento no constituye un problema para el veritismo.

Una epistemología veritista no niega que haya unidades objeto de evaluación epistémica distintas de la creencia (tales como métodos, prácticas y rasgos de sujetos), solamente sostiene que la evaluación distintivamente epistémica de esas unidades debe hacer referencia de manera esencial a alguna conexión apropiada que ellas tengan con el valor de la creencia verdadera. Las habilidades cognitivas son unidades que pueden evaluarse desde ese punto de vista veritista de la siguiente manera. Las habilidades cognitivas que se piensa que están constitutivamente asociadas con estados de entendimiento son habilidades para predecir, diagnosticar, imaginar y explicar. El ejercicio exitoso de cualquiera de estas habilidades da lugar a los resultados canónicos de tales habilidades, es decir, buenas predicciones, buenos diagnósticos, buenas descripciones contrafácticas y buenas explicaciones. Ahora bien, lo que hace que cualquiera de estos resultados canónicos de las habilidades sea epistémicamente bueno es que de alguna manera cumple con un estándar veritista de evaluación. Por ejemplo, lo que hace que una predicción sea epistémicamente buena es (por lo menos) que se realiza a partir de evidencias que hacen que la probabilidad de que resulte verdadera sea alta. Lo que hace que una descripción contrafáctica sea epistémicamente buena es (por lo menos) que la probabilidad de que su consecuente sea verdadero es alta dada la hipótesis de que su antecedente lo es. Así pues, podemos conjeturar que el estándar de corrección de la creencia, i.e., la verdad, se aplica a los resultados canónicos de las habilidades en cuestión; tales resultados tienen valor epistémico en la medida en que satisfacen ese estándar. Puesto que cuando una de esas habilidades cognitivas sistemáticamente produce resultados fallidos -i.e., predicciones de baja probabilidad, descripciones contrafácticas incorrectas, etc.- se evalúa negativamente, resulta razonable suponer que atribuimos valor epistémico a esas habilidades sólo en la medida en que producen resultados que consideramos epistémicamen- 
te valiosos; las habilidades tienen valor epistémico porque sus resultados lo tienen. De este modo, el valor epistémico de las habilidades parecería ser un tipo de valor instrumental en relación con el valor epistémico de sus resultados canónicos.

Por mor del argumento concedimos al objetor del veritismo que el valor epistémico de las habilidades constitutivamente asociadas con estados de entendimiento no puede explicarse asumiendo que la posesión de esas habilidades sea equivalente a, o explicable en términos de, estados de conocimiento proposicional, $\mathrm{u}$ otros estados proposicionales atribuibles al sujeto de las habilidades. Pero el valor epistémico de esas habilidades todavía puede explicarse en términos veritistas como una especie de valor que es instrumental en relación con el valor de sus resultados canónicos - tales como predicciones de alta probabilidad o descripciones contrafácticas correctas- cuyo valor epistémico se determina aplicándoles, de la manera indicada en el párrafo anterior, el estándar veritista de la verdad.

Esta respuesta al aparente problema planteado por el valor epistémico de las habilidades asociadas con estados de entendimiento da por sentado que el valor epistémico de las habilidades es instrumental, que tienen valor epistémico en virtud del valor epistémico de sus resultados canónicos. Los oponentes del veritismo podrían disputar esta suposición instrumentalista, podrían argumentar que se pueden obtener los resultados de las habilidades sin poseer genuinamente la habilidad; por ejemplo, alguien que por mero azar, o cometiendo una serie de errores que terminan por cancelarse entre sí, llega a predicciones que de hecho tienen alta probabilidad dadas las evidencias. Pero lograr los resultados por medio de la habilidad —argumentarían- es epistémicamente más valioso que lograrlos de alguna otra manera; por lo tanto, poseer la habilidad es epistémicamente valioso por algo más que el mero hecho de que la habilidad entregue sus resultados canónicos. ¿Cómo podría una epistemología veritista dar cuenta de ese valor epistémico no instrumental implicado en el ejercicio de habilidades cognitivas?

Para responder esta pregunta debemos notar que el valor epistémico adicional asociado a la posesión de la habilidad deriva del hecho de que un ejercicio genuino de la habilidad hace posible que sus resultados sean acreditables al agente o atribuibles a él como sus logros propios, lo cual no ocurre cuando los resultados se alcanzan de una manera ajena al ejercicio genuino de la habilidad; por ejemplo, por mero azar o cometiendo una serie de errores. Este tipo de valor es precisamente la clase de valor asociado con el mérito epistémico que mencionamos en la sección 1 de este trabajo como ejemplo de un valor epistémico 
que una epistemología veritista tolerante, pero no una dura, puede explicar. Esto significa que, aun cuando el objetor tenga razón sobre la existencia de un valor epistémico asociado al ejercicio de las habilidades cognitivas que no es instrumental con respecto al valor epistémico de los resultados canónicos de esas habilidades, de ello no se sigue que el veritista no pueda dar cuenta de tal valor. Como señalé al inicio de este trabajo, las epistemologías veritistas tolerantes poseen los recursos conceptuales para explicar el valor epistémico de ese tipo de mérito. Lo que hace que el valor del mérito implícito en lograr una predicción de alta probabilidad - o una descripción contrafáctica correcta- mediante habilidades cognitivas propias sea un valor epistémico no es nada en la estructura de evaluación que hace posible la acreditación de esos logros al sujeto de las habilidades, pues la misma estructura está presente en otros ámbitos no epistémicos cumpliendo la misma función de hacer acreditable un bien al sujeto. Por ejemplo, en el ámbito estético una bailarina logra una ejecución magistral gracias a habilidades dancísticas propias, lo cual hace posible que tal ejecución se le acredite a ella como un logro de mérito propio. ${ }^{15}$ Asimismo, en el ámbito deportivo, un futbolista logra un gol espectacular gracias a habilidades atléticas propias, lo cual hace posible que tal gol se le acredite a él como un logro de mérito propio. En ninguno de estos dos casos el mérito del logro alcanzado mediante el ejercicio de habilidades propias es distintivamente epistémico. Lo que hace que el mérito de lograr una predicción o una descripción correctas mediante el ejercicio de habilidades cognitivas propias sea distintivamente epistémico es que esos resultados canónicos de la habilidades implicadas satisfacen el estándar de la verdad. De este modo, la explicación del valor distintivamente epistémico del mérito implícito en el ejercicio exitoso de las habilidades cognitivas constitutivamente asociadas con el entendimiento es explicable en el marco de una epistemología veritista tolerante haciendo referencia esencial al valor de la creencia verdadera, sin tener que asumir que el valor epistémico de ese mérito sea meramente instrumental en relación con el valor de la creencia verdadera.

\section{Conclusiones}

La defensa del veritismo que se ha presentado en este trabajo ante a la acusación de que no puede explicar la naturaleza o el valor del entendi-

${ }^{15}$ El ejemplo de la evaluación de la ejecución de una bailarina es de Sosa 2007, cap. 4.

Diánoia, vol. LV, no. 65 (Noviembre 2010). 
miento temático tiene un alcance limitado. El propósito del trabajo no ha sido mostrar que no haya nada en la naturaleza o el valor epistémico del entendimiento que sea problemático para el veritismo, sino simplemente que las objeciones aquí consideradas no consiguen mostrar que el veritismo sufra déficits explicativos. Aunque las cuatro objeciones examinadas fracasen por las razones expuestas en este artículo, ello es compatible con que exista alguna otra objeción al veritismo basada en la naturaleza o el valor del entendimiento que represente un genuino problema para la epistemología veritista. Investigar si existe un problema tal es tema para otro trabajo.

Pero considero que los resultados obtenidos en la discusión de las objeciones aquí examinadas son instructivos con vistas al examen de otras potenciales objeciones al veritismo. La idea, discutida en la sección 2 , de que hay algo sui generis en los estados de apreciación o captación de la realidad propios del entendimiento es difícil de sostener porque cualquiera de las dos maneras de interpretar esos estados, como fácticos o como meramente dóxicos, hace que no sean problemáticos para la evaluación veritista. Por otro lado, vimos en la sección 3 que el hecho de que el entendimiento pueda lograrse mediante vehículos cuya estructura no es proposicional no implica que los estados mismos de entendimiento no tengan contenido proposicional. De modo que si no se da un argumento adicional, el hecho de que en la génesis de un estado de entendimiento se encuentren vehículos no proposicionales no puede tomarse como evidencia de que el objeto del entendimiento en esos casos no sea proposicional sino algo sui generis. En lo que se refiere al valor epistémico del entendimiento, vimos en la sección 4 que al examinar aspectos valiosos del entendimiento debemos determinar, en primer lugar, si hay razón para pensar que el valor en cuestión sea epistémico, pues si no lo es, entonces no impone al veritismo, ni a cualquier teoría de la evaluación distintivamente epistémica, ninguna demanda explicativa. Virtudes que hacen valioso entender algo, como la organización y la sistematicidad, poseen un valor epistémico cuya explicación no es problemática para el veritismo, y aunque esas virtudes poseen otros tipos de valor, hay razón para pensar que no se trata de valores epistémicos. En la sección 5 vimos que otro aspecto que hace valioso el entendimiento es que se encuentra asociado con ciertas habilidades cognitivas; éstas poseen un valor epistémico (instrumental) que incluso el veritismo duro puede explicar. Sin embargo, también poseen un valor de mérito que no es instrumental. Expuse cómo este tipo de valor epistémico puede explicarlo una teoría veritista tolerante que respete la idea más básica del veritismo: el valor de la creencia verdadera

Diánoia, vol. LV, no. 65 (Noviembre 2010). 
está de alguna manera esencial involucrado en la explicación de todo valor epistémico. Los objetores del veritismo parecen subestimar la fexibilidad explicativa de éste núcleo, el más básico, de las epistemologías veritistas.

\section{BIBLIOGRAFÍA}

DePaul, M. y S. Grimm, 2007, "Review Essay on Jonathan Kvanvig's The Value of Knowledge and the Pursuit of Understanding", Philosophy and Phenomenological Research, vol. 74, no. 2, pp. 498-514.

DePaul, M. y L. Zagzebski, (comps.), 2003, Intellectual Virtue: Perspectives from Ethics and Epistemology, Oxford University Press, Oxford.

Goldman, A., 2001, "The Unity of Epistemic Virtues", en A. Fairweather y L. Zagzebski (comps.), Virtue Epistemology. Essays on Epistemic Virtue and Responsibility, Oxford University Press, Oxford.

_ 1999, Knowledge in a Social World, Oxford University Press, Oxford.

_ 1976, "Discrimination and Perceptual Knowledge", Journal of Philosophy. vol. 73, no. 20, pp. 771-791.

Greco, J., 2010, Achieving Knowledge. A Virtue-Theoretic Account of Epistemic Evaluation, Cambridge University Press, Cambridge.

—, 2003 , "Knowledge as Credit for True Belief", en DePaul y Zagzebski 2003, pp. 111-134.

Grimm, S., 2006, "Is Understanding a Species of Knowledge?", The British Journal for the Philosophy of Science, vol. 57, no. 3, pp. 515-535.

Kvanvig, J., 2003, The Value of Knowledge and the Pursuit of Understanding, Cambridge University Press, Nueva York.

Pritchard, D., 2010, "The Value Problem for Knowledge", en A. Haddock, A. Millar y D.H. Pritchard, The Nature and Value of Knowledge: Three Investigations, Oxford University Press, Oxford.

Riggs, W., 2003, "Understanding Virtue and the Virtue of Understanding", en DePaul y Zagzebski 2003, pp. 203-226.

- 2002, "Beyond Truth and Falsehood: The Real Value of Knowing that $p$ ", Philosophical Studies, vol. 107, no. 1, pp. 87-108.

Sosa, E., 2007, A Virtue Epistemology. Apt Belief and Reflective Knowledge, vol. I, Oxford University Press, Oxford.

_ 2003, "The Place of Truth in Epistemology", en DePaul y Zagzebski 2003, pp. $155-180$.

Williamson, T., 2000, Knowledge and Its Limits, Oxford University Press, Oxford. Woodward, J., 2003, Making Things Happen: A Theory of Causal Explanation, Oxford University Press, Nueva York.

Zagzebski, L., 2001, "Recovering Understanding", en M. Steup (comp.), Knowledge, Truth and Duty. Essays on Epistemic Justification, Responsibility, and Virtue, Oxford University Press, Oxford, pp. 235-252.

Recibido el 26 de noviembre de 2009; aceptado el 2 de agosto de 2010. 